\title{
AN UNDERSTANDING OF THE SHOULDER OF GIANTS: JOVIAN PLANETS AROUND LATE K DWARF STARS AND THE TREND WITH STELLAR MASS
}

\author{
Eric Gaidos ${ }^{1}$, Debra A. Fischer ${ }^{2}$, Andrew W. Mann ${ }^{3}$, And Andrew W. Howard ${ }^{3}$ \\ ${ }^{1}$ Department of Geology and Geophysics, University of Hawai'i at Mānoa, Honolulu, HI 96822, USA; gaidos@ hawaii.edu \\ 2 Department of Astronomy, Yale University, New Haven, CT 06520, USA \\ ${ }^{3}$ Institute for Astronomy, University of Hawai 'i at Mānoa, Honolulu, HI 96822, USA \\ Received 2013 April 17; accepted 2013 May 11; published 2013 June 11
}

\begin{abstract}
Analyses of exoplanet statistics suggest a trend of giant planet occurrence with host star mass, a clue to how planets like Jupiter form. One missing piece of the puzzle is the occurrence around late $\mathrm{K}$ dwarf stars (masses of $0.5-0.75 M_{\odot}$ and effective temperatures of 3900-4800 K). We analyzed four years of Doppler radial velocity (RVs) data for 110 late $\mathrm{K}$ dwarfs, one of which hosts two previously reported giant planets. We estimate that $4.0 \% \pm 2.3 \%$ of these stars have Saturn-mass or larger planets with orbital periods $<245$ days, depending on the planet mass distribution and RV variability of stars without giant planets. We also estimate that $0.7 \% \pm 0.5 \%$ of similar stars observed by Kepler have giant planets. This Kepler rate is significantly ( $99 \%$ confidence) lower than that derived from our Doppler survey, but the difference vanishes if only the single Doppler system (HIP 57274) with completely resolved orbits is considered. The difference could also be explained by the exclusion of close binaries (without giant planets) from the Doppler but not Kepler surveys, the effect of long-period companions and stellar noise on the Doppler data, or an intrinsic difference between the two populations. Our estimates for late K dwarfs bridge those for solar-type stars and M dwarfs, and support a positive trend with stellar mass. Small sample size precludes statements about finer structure, e.g., a "shoulder" in the distribution of giant planets with stellar mass. Future surveys such as the Next Generation Transit Survey and the Transiting Exoplanet Satellite Survey will ameliorate this deficiency.
\end{abstract}

Key words: astrobiology - planetary systems - planets and satellites: formation - stars: fundamental parameters techniques: radial velocities

Online-only material: color figure

\section{INTRODUCTION}

Parent star mass is a fundamental parameter in planet discovery space because there are both theoretical predictions and observational evidence that the properties of planetary systems depend on central star mass. In addition, it is of practical significance: the sensitivity of the Doppler, astrometric, and transit techniques of planet detection scale inversely with stellar mass (or radius), such that smaller planets can be detected around less massive stars (or smaller stars).

Most surveys for planets, i.e., ground-based radial velocity (RV) surveys and the Kepler transit survey mission, are flux- or magnitude-limited at visible wavelengths, favoring the inclusion of intrinsically bright stars. In addition, the Doppler method only works with spectra having large numbers of deep absorption lines, which disqualifies hot stars, and the transit method works better for cool dwarfs, around which planets produce deeper transits. These opposing trends mean that catalogs of exoplanethosting stars are dominated by solar-type stars with late $\mathrm{F}$ to early K spectral types (Udry et al. 2007; Batalha et al. 2010). Moreover, a focus on solar-mass stars satisfies a desire to determine the occurrence, nature, and potential habitability of planets around other stars like the Sun.

On the other hand, $\mathrm{M}$ dwarf stars are now widely recognized as an attractive "short cut" to the discovery of Earth-like planets because such stars are numerous, small, and their habitable zones are close-in, meaning that planets orbiting within them will be more detectable by the Doppler or transit methods. Doppler surveys have included the few nearby $M$ dwarfs that are sufficiently bright at visible wavelengths (Bonfils et al. 2013), and high-precision infrared spectrographs are being constructed to take advantage of the greater emission of these stars at longer wavelengths (Artigau et al. 2011; Quirrenbach et al. 2012). Several thousand M dwarfs were added to the Kepler target catalog for these reasons (Batalha et al. 2010).

Between the early-K-type dwarfs and $\mathrm{M}$ dwarfs are the late $\mathrm{K}$ dwarfs, having $\mathrm{K} 4-\mathrm{K} 7$ spectral subtypes, $T_{\text {eff }} \approx$ $3900-4800 \mathrm{~K}$, and $M_{*} \approx 0.5-0.75 M_{\odot}$. These stars have been comparatively neglected in planet surveys because they are intrinsically faint and they are not $\mathrm{M}$ dwarfs. The two largest Doppler surveys, the California Planet Search (CPS) and the HARPS survey, include relatively few late K stars. Ironically, these stars may be especially attractive targets for Doppler surveys because intrinsic stellar Doppler noise or "jitter" decreases with later spectral type and could be $<1 \mathrm{~m} \mathrm{~s}^{-1}$ among $\mathrm{K}$ dwarfs (Isaacson \& Fischer 2010; Lovis et al. 2011). The K5 dwarf HD 85512 is one of the most Doppler-stable stars reported (residual $\mathrm{rms}=0.75 \mathrm{~m} \mathrm{~s}^{-1}$ ), a property that has permitted the discovery of a super-Earth near or inside its habitable zone (Pepe et al. 2011).

Giant planets, defined here as planets with mass greater than that of Saturn $\left(95 M_{\oplus}\right)$ or radius greater than $8 R_{\oplus}$, are readily detected by Doppler observations with a sufficient time baseline if the planets orbit within $\sim 1 \mathrm{AU}$ of their host stars. Their distribution with mass or spectral type of the host star can test the core accretion scenario of giant planet formation as well as models of orbital migration. Numerous studies have found evidence that the fraction of stars with giant planets increases with stellar mass (Fischer \& Valenti 2005; Cumming et al. 2008; Johnson et al. 2010). Likewise, Fressin et al. (2013) estimated that the occurrence of giant planets is lower for Kepler M dwarfs 
Table 1

Confirmed Giant Planets around Mid- and Late-K-type Dwarf Stars ${ }^{\mathrm{a}}$

\begin{tabular}{|c|c|c|c|c|c|c|}
\hline Planet & $\begin{array}{c}\text { Mass } \\
\left(M_{J}\right)\end{array}$ & $\begin{array}{l}\text { Period } \\
\text { (days) }\end{array}$ & SpT & $B-V$ & $T_{\text {eff }}$ & References \\
\hline WASP-80b & 0.55 & 3.068 & K7-M0 & 0.94 & $\sim 4000$ & Triaud et al. (2013) \\
\hline HIP $70849^{b}$ & $>3$ & $>5 \mathrm{yr}$ & $\mathrm{K} 7$ & 1.42 & $4100^{c}$ & Ségransan et al. (2011) \\
\hline WASP-43b & 2.03 & 0.813 & K7 & 1.0 & $4400^{\mathrm{d}}$ & Hellier et al. (2011) \\
\hline HAT-P-20b & 7.25 & 2.88 & K3 & $\cdots$ & 4619 & Bakos et al. (2011a); Torres et al. (2012) \\
\hline HIP $57274 \mathrm{c}$ & $0.41^{\mathrm{e}}$ & 32.0 & K4 & 1.11 & 4640 & Fischer et al. (2012) \\
\hline HIP 57274d & $0.53^{\mathrm{e}}$ & 431.7 & & & & \\
\hline WASP-59b & 0.0 .86 & 7.92 & K5 & 0.92 & $4650^{\mathrm{h}}$ & Hébrard et al. (2013) \\
\hline HD $113538 b$ & $0.27^{\mathrm{e}}$ & 263.3 & $K 9^{f}$ & 1.38 & 4685 & Moutou et al. (2011) \\
\hline HD $113585 \mathrm{c}$ & $0.71^{\mathrm{e}}$ & 1657 & & & & \\
\hline HIP 2247b & $5.12^{\mathrm{e}}$ & 655.6 & K4 & 1.14 & 4714 & Moutou et al. (2009) \\
\hline WASP-10b & 3.06 & 3.09 & K5 & $\ldots$ & 4735 & Christian et al. (2009); Torres et al. (2012) \\
\hline BD -08 2823b & 0.33 & 237.6 & K3 & 1.07 & 4746 & Hébrard et al. (2010) \\
\hline HD $20868 b$ & 1.99 & 380.85 & $\mathrm{~K} 3 / 4$ & 1.04 & 4795 & Moutou et al. (2009) \\
\hline HD $63454 b$ & $0.38^{\mathrm{e}}$ & 2.82 & K4 & 1.06 & 4840 & Moutou et al. (2005) \\
\hline Qatar-1b & 1.09 & 1.42 & $\mathrm{~N} / \mathrm{A}$ & 1.06 & 4861 & Alsubai et al. (2011) \\
\hline HIP $5158 b^{g}$ & $1.44^{\mathrm{e}}$ & 345.6 & K5 & 1.08 & 4962 & Lo Curto et al. (2010) \\
\hline
\end{tabular}

\footnotetext{
Notes.

${ }^{\text {a }} M_{p} \sin i>0.3 M_{J}$ and $\mathrm{K}$ dwarf hosts with spectral subtypes 4 or later in the Exoplanet Catalog (Schneider et al. 2011$)$ or $1<B-V<1.5$ in the Exoplanets Data Explorer (Wright et al. 2011).

$\mathrm{b}$ Incomplete orbit and parameters are poorly constrained.

${ }^{c}$ Based on infrared photometry and the temperature-luminosity relation of Baraffe et al. (1998).

${ }^{\mathrm{d}} T_{\text {eff }}$ based on $\mathrm{H} \alpha$ and may need to be revised upward based on a new mass estimate (Gillon et al. 2012).

e $M_{P} \sin i$.

${ }^{\mathrm{f}}$ Stellar parameters are problematic: Gray et al. (2006) assign it the unrecognized spectral type K9, and its $B-V$ and $V-J$ colors suggest a star at the K-M spectral type boundary. Gray et al. (2006) also assign it a " $\mathrm{k}$ " to indicate interstellar absorption features, seemingly inconsistent for a star only $16 \mathrm{pc}$ away. Moutou et al. (2011) and Bailer-Jones (2011) assign $T_{\text {eff }}$ of $4685 \mathrm{~K}$ and $4625 \mathrm{~K}$ based on spectra and photometry, respectively. To reconcile the $T_{\text {eff }}$ and colors, Bailer-Jones (2011) estimate $\sim 1$ mag of extinction, also inconsistent with its proximity.

$\mathrm{g}$ This system also includes HIP 5185c, which may be a brown dwarf (Feroz et al. 2011).

${ }^{\mathrm{h}} T_{\text {eff }}$ was estimated by the null dependence of abundance on excitation potential.
}

than for $\mathrm{G}$ and $\mathrm{K}$ dwarfs. These findings support a theoretical prejudice that a more massive star is born with a more massive disk that can spawn the solid cores capable of accreting disk gas before its dispersal (Laughlin et al. 2004). However, any relation between disk mass and stellar mass is ambiguous (Williams \& Cieza 2011). Moreover, the picture below $M_{*}=0.75 M_{\odot}$ is unclear because the statistics are poor; the sample of Johnson et al. (2010) included only 142 late $\mathrm{K}$ and $\mathrm{M}$ dwarfs with 5 reported giant planets. Likewise, the difference in giant planet occurrence around solar-type and $\mathrm{M}$ dwarf stars reported by Fressin et al. (2013) has only a $1.3 \sigma$ significance. K dwarfs provide the "missing link" in this picture, and surveys for giant planets could reveal whether the difference in giant planet frequency exists, and whether it is a smooth transition or an abrupt "shoulder."

The M2K Doppler survey targets the brightest late K dwarfs, bridging the gap between solar-type stars and $\mathrm{M}$ dwarfs. The survey reported one giant planet around an M3 dwarf (Apps et al. 2010) and a triple system including two Saturn- to Jupitermass planets around the K4-K5 dwarf HIP 57274 (GJ 439) with $T_{\text {eff }} \approx 4640 \pm 100 \mathrm{~K}$ and $M_{*} \approx 0.73 \pm 0.05$, based on Yale-Yonsei isochrones (Fischer et al. 2012). Only four other mid- to late-K-type dwarf hosts of giant planets have been reported: Jupiter-mass HAT-20b transits a similar K3 star with $T_{\text {eff }} \approx 4619 \pm 72 \mathrm{~K}$ (Bakos et al. 2011b; Torres et al. 2012). The effective temperatures of the other three late-K-type hosts (WASP-43b, HIP 70849, and WASP-80b) are not well established (Table 1 and Section 4.4). All other K dwarf hosts are hotter and have earlier spectral subtypes. We use the results of the M2K survey and Kepler to establish new constraints on the occurrence of giant planets around late $\mathrm{K}$ dwarfs and compare them with values for solar-type stars and M dwarfs.

\section{METHODS: M2K SURVEY}

\subsection{Sample, Observations, and Reduction}

Doppler observations were performed with the High Resolution Echelle Spectrograph (HIRES) on the Keck I telescope (Vogt et al. 1994). Observations obtained $R=55,000$ with the B5 decker and a typical S/N of 200. Wavelength calibration was provided by a molecular iodine cell in the beam line. RV solutions were obtained by a forward modeling process in which an intrinsic stellar spectrum is obtained without the iodine cell, multiplied by an $R \sim 5 \times 10^{5}$ spectrum of the iodine cell taken with a Fourier transform spectrograph, and convolved by the instrumental profile. The relative shift in wavelength between the model and observed spectra is a free parameter. The median formal measurement error in the $\mathrm{M} 2 \mathrm{~K}$ survey is $1.25 \mathrm{~m} \mathrm{~s}^{-1}$. We obtained $N=4$ or more RV measurements on 159 stars. The median number of measurements for each star used in our analysis is $N=9$, however the distribution of number of measurements is very uneven because of our strategy of follow-up of RV-variable stars (Figure 1).

Ideally, we would have defined our sample of late K dwarfs in terms of effective temperature $T_{\text {eff }}$, a fundamental stellar parameter, but not all our stars have spectroscopically-determined values. Thus, we selected stars based on $V-J$ color as a proxy for $T_{\text {eff }}$. We restricted the sample to 140 stars with $1.8<V-J<2.8$, corresponding to $3900<T_{\text {eff }}<4750 \mathrm{~K}$ or spectral subtypes K3-K7 (Gray \& Corbally 2009), based on 


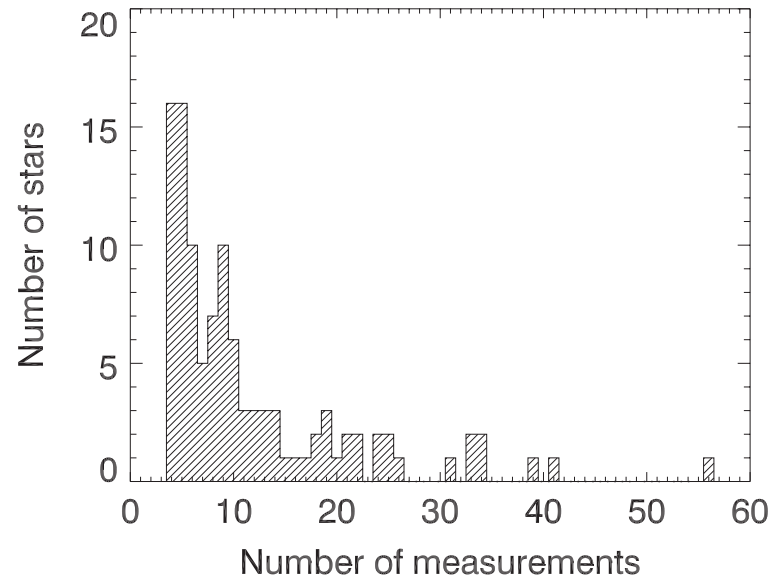

Figure 1. Distribution of number of Doppler measurements per star for those M2K stars selected for the analysis of giant planet fraction. One star (HIP 57274) with two giant planets has 120 observations and is off scale.

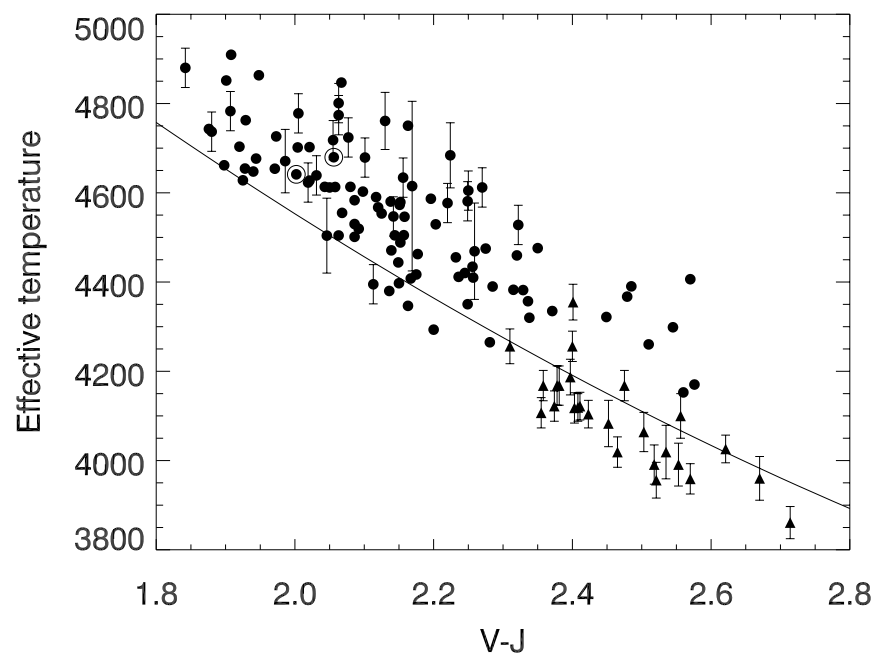

Figure 2. Stellar effective temperature vs. $V-J$ color of late $\mathrm{K}$ dwarfs in the M2K Doppler survey. Circles represent temperatures from SME analyses of high-resolution spectra (Valenti \& Piskunov 1996), whereas triangles represent temperatures from fitting medium-resolution spectra to PHOENIX synthetic spectra (A. W. Mann et al. 2013, in preparation) and calibrating on stars in Boyajian et al. (2012). Only some error bars are shown for clarity. The solid curve is an empirical $T_{\text {eff }}$ vs. $V-J$ relation from Boyajian et al. (2012). Two systems with published giant planets (HIP 57274 and HIP 2247) are circled.

an empirical color- $T_{\text {eff }}$ relation using stars with measured angular diameters (Boyajian et al. 2012). We corroborated this selection by estimating the $T_{\text {eff }}$ of many of these stars using spectra (Figure 2). Stellar parameters (including $T_{\text {eff }}$ and metallicity $[\mathrm{Fe} / \mathrm{H}])$ of stars with $1.8<V-J<2.3$ were estimated using the Spectroscopy Made Easy (SME) package (Valenti \& Piskunov 1996; Table 2). SME performs poorly on dwarfs with $V-J>2.3$ and $T_{\text {eff }} \leqslant 4300 \mathrm{~K}$ so for these stars we estimated $T_{\text {eff }}$ by comparing moderate-resolution $(R \sim 1500)$ visible-wavelength (3500-8500 $\AA$ ) spectra obtained with the Supernova Integral Field Spectrograph (Lantz et al. 2004) on the University of Hawaii $2.2 \mathrm{~m}$ telescope with synthetic spectra generated by the PHOENIX BT-SETTL program (Allard et al. 2011). The comparison procedure is described in Lépine et al. (2013) and was adjusted (A. W. Mann et al. 2013, in preparation) to maximize agreement with the calibrator stars of Boyajian et al. (2012). For stars without spectra, we estimated $T_{\text {eff }}$ using $V-J$ color and the Boyajian et al. (2012) relation (Table 2).

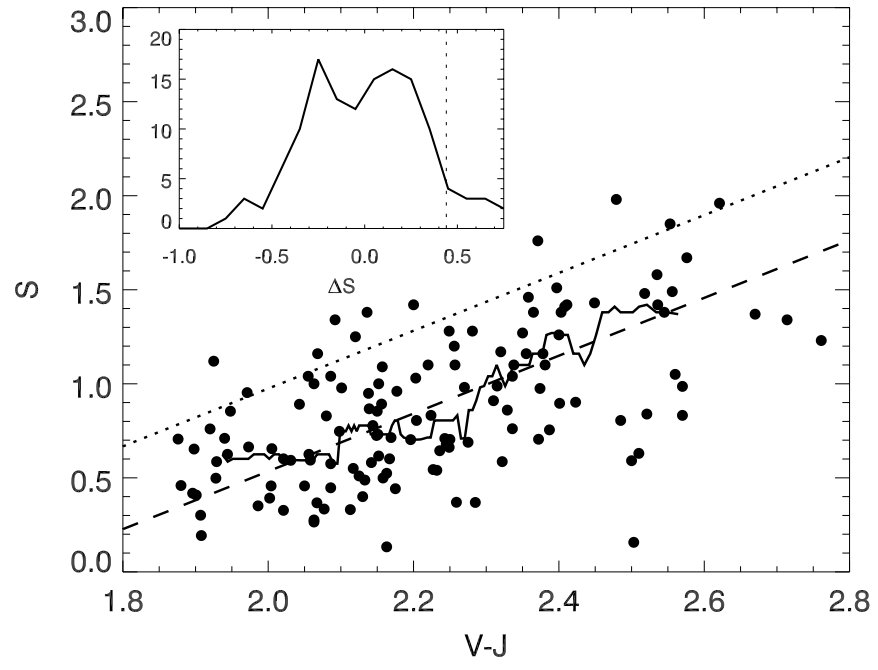

Figure 3. Flux $S$ in the core of the Ca II HK lines, normalized by the continuum, vs. $V-J$ color. High values of $S$ are associated with elevated stellar activity and astrophysical Doppler noise or "jitter." The solid line is a running median $(N=20)$, the dashed line is a linear regression of the median $\bar{S}$, and the dotted line is the linear fit +0.44 , above which stars were excluded from the analysis. This threshold was selected based on the distribution of $\Delta S=S-\bar{S}$ (inset). Eleven stars or $8 \%$ of the sample were excluded based on this criterion.

The median offset between SME-derived and $V-J$-based temperatures is $140 \mathrm{~K}$. For this reason, we included the 13 stars with SME-based $T_{\text {eff }}>4750 \mathrm{~K}$ (but acceptable $V-J$ ) as their actual effective temperatures are probably within the acceptable range. Unsurprisingly, $T_{\text {eff }}$ estimates based on calibrated comparisons between moderate-resolution spectra and PHOENIX models are consistent with the Boyajian et al. (2012) relation. Nearly all of our stars have measured parallaxes and we estimated masses using the relation with absolute $K$ magnitude in Henry \& McCarthy (1993). For those few stars lacking parallaxes we used the empirical relations between $T_{\text {eff }}$, stellar radius, and stellar mass in Boyajian et al. (2012).

We excluded stars exhibiting relatively high emission in the $\mathrm{H}$ and $\mathrm{K}$ lines of $\mathrm{Ca}$ II. These stars are chromospherically active and tend to exhibit higher astrophysical Doppler noise or "jitter" (e.g., Isaacson \& Fischer 2010). Figure 3 shows values of $S_{\mathrm{HK}}$, the flux in the Ca II line cores normalized by the continuum, versus $V-J$ color. The trend of increasing $S_{\mathrm{HK}}$ with redder $V-J$ is due to the lower blue continuum rather than elevated $\mathrm{Ca}$ II emission in redder stars. We calculated a running median $(N=20)$, fit a linear function $\bar{S}_{\mathrm{HK}}$ with $V-J$, and subtracted that from these values. The histogram (inset of Figure 3) suggests a cutoff at $S_{\mathrm{HK}}-\bar{S}_{\mathrm{HK}}=0.44$, which rejects 11 stars as exceptionally active. Among the stars we admitted were five for which it was not possible to estimate $S_{\mathrm{HK}}$ because the continuum was not detected.

We next considered the distribution of RV standard deviations (rms) of the remaining 129 stars. The majority (100) of the stars fall in a cluster with rms $<15 \mathrm{~m} \mathrm{~s}^{-1}$ (Figure 4). We inspected the RV data of the 29 stars with rms $>15 \mathrm{~m} \mathrm{~s}^{-1}$. One of these is HIP 57274, which has been described elsewhere (Fischer et al. 2012). Eight others have additional stars within 5 arcsec and were excluded because leakage of light into the spectrograph slit is an established source of RV error.

We analyzed each of the remaining 20 sets of RVs with both weighted linear and quadratic regressions and applied an $F$-test to evaluate the significance of any reduction in variation after subtraction of the fit. Although all stars have $\geqslant 4 \mathrm{RV}$ 
Table 2

Stars Included in the Analysis

\begin{tabular}{|c|c|c|c|c|c|c|c|c|}
\hline Name & $V-J$ & $T_{\text {eff }}(\mathrm{K})$ & $(\mathrm{Fe} / \mathrm{H})$ & $S$ & $M\left(M_{\odot}\right)$ & $N_{\text {obs }}$ & $\mathrm{rms}\left(\mathrm{m} \mathrm{s}^{-1}\right)$ & $p^{1}$ \\
\hline HIP 1078 & 2.13 & $4426^{\mathrm{b}}$ & $\ldots$ & 0.49 & 0.75 & 4 & 2.56 & 0.005 \\
\hline HIP 1532 & 2.52 & $3956^{\mathrm{a}}$ & -0.37 & 0.84 & 0.64 & 24 & 4.28 & 0.011 \\
\hline HIP 2247 & 2.06 & 4680 & 0.24 & 0.62 & 0.77 & 12 & 0.00 & 0.000 \\
\hline HIP 3418 & 2.16 & 4546 & 0.02 & 0.50 & 0.73 & 5 & 1.54 & 0.004 \\
\hline HIP 4353 & 2.20 & 4587 & 0.17 & 0.70 & 0.77 & 6 & 12.89 & 0.961 \\
\hline HIP 4454 & 1.99 & 4671 & -0.55 & 0.35 & 0.71 & 5 & 3.62 & 0.004 \\
\hline HIP 4845 & 2.58 & 4170 & -0.19 & 1.67 & 0.63 & 33 & 3.55 & 0.005 \\
\hline HIP 5247 & 2.51 & 4260 & -0.22 & 0.63 & 0.66 & 12 & 7.76 & 0.052 \\
\hline HIP 5663 & 2.34 & 4357 & -0.05 & 1.04 & 0.68 & 24 & 0.00 & 0.000 \\
\hline HIP 6344 & 2.32 & 4383 & -0.04 & 0.99 & 0.68 & 5 & 1.71 & 0.007 \\
\hline HIP 9788 & 2.15 & 4444 & -0.37 & 0.73 & 0.67 & 17 & 4.53 & 0.008 \\
\hline HIP 10337 & 2.54 & $4019^{a}$ & 0.12 & 1.58 & 0.66 & 22 & 7.74 & 0.019 \\
\hline HIP 10416 & 1.88 & 4743 & 0.14 & 0.71 & 0.76 & 11 & 10.43 & 0.721 \\
\hline HIP 11000 & 1.92 & 4703 & 0.20 & 0.76 & 0.73 & 13 & 10.54 & 0.195 \\
\hline HIP 12493 & 2.25 & 4350 & -0.29 & 0.66 & 0.68 & 6 & 4.50 & 0.032 \\
\hline HIP 13375 & 2.50 & $4110^{\mathrm{b}}$ & 0.00 & 0.59 & 0.56 & 9 & 3.68 & 0.010 \\
\hline HIP 14729 & 2.15 & 4579 & 0.17 & 1.00 & 0.70 & 10 & $5.76^{\mathrm{c}}$ & 0.007 \\
\hline HIP 15095 & 2.28 & 4265 & -0.21 & 1.28 & 0.68 & 10 & 6.82 & 0.071 \\
\hline HIP 15563 & 2.06 & 4718 & 0.16 & 1.04 & 0.72 & 12 & 10.74 & 0.332 \\
\hline HIP 15673 & 1.93 & 4654 & -0.46 & 0.50 & 0.69 & 6 & 3.12 & 0.007 \\
\hline TYC 1234-00069-1 & 1.90 & $4522^{\mathrm{a}}$ & $\ldots$ & 0.42 & 0.75 & 4 & 1.30 & 0.023 \\
\hline HIP 17346 & 2.06 & 4613 & 0.07 & 0.59 & 0.74 & 6 & 4.19 & 0.012 \\
\hline HIP 17496 & 2.15 & 4489 & -0.04 & 0.62 & 0.72 & 5 & 2.53 & 0.008 \\
\hline HIP 19165 & 2.24 & 4412 & -0.22 & 0.64 & 0.65 & 31 & 5.16 & 0.010 \\
\hline HIP 19981 & 2.25 & 4605 & 0.27 & 0.70 & 0.77 & 5 & 4.60 & 0.017 \\
\hline HIP 20359 & 2.14 & 4547 & -0.04 & 0.58 & 0.75 & 5 & 4.47 & 0.011 \\
\hline HIP 25220 & 2.04 & 4613 & 0.05 & 0.89 & 0.71 & 10 & 7.92 & 0.049 \\
\hline HIP 26196 & 2.34 & $4245^{\mathrm{b}}$ & $\ldots$ & 0.76 & 0.74 & 8 & $4.26^{\mathrm{d}}$ & 0.012 \\
\hline TYC 4356-01014-1 & 1.88 & 4737 & -0.17 & 0.46 & 0.77 & 8 & 2.69 & 0.003 \\
\hline HIP 29548 & 2.32 & 4528 & -0.10 & 0.59 & 0.69 & 20 & 3.79 & 0.006 \\
\hline HIP 30112 & 2.36 & $4168^{\mathrm{a}}$ & $\cdots$ & 1.46 & 0.72 & 25 & 7.45 & 0.040 \\
\hline HIP 30979 & 2.02 & 4627 & 0.23 & 0.60 & 0.77 & 12 & 5.41 & 0.005 \\
\hline TYC 3388-01009-1 & 2.36 & $4220^{\mathrm{b}}$ & $\ldots$ & 1.38 & 0.68 & 10 & 64.66 & 0.500 \\
\hline HIP 32769 & 2.24 & 4420 & -0.05 & 0.68 & 0.71 & 16 & 4.86 & 0.005 \\
\hline HIP 32919 & 2.33 & 4382 & -0.01 & 0.86 & 0.70 & 18 & 5.29 & 0.006 \\
\hline TYC 1352-01588-1 & 2.31 & $4270^{\mathrm{b}}$ & $\ldots$ & 0.00 & 0.69 & 7 & 18.27 & 0.918 \\
\hline TYC 0748-01711-1 & 2.50 & $4064^{\mathrm{a}}$ & $\cdots$ & 0.16 & 0.66 & 25 & $42.28^{c}$ & 0.500 \\
\hline HIP 36551 & 2.09 & 4501 & -0.30 & 0.57 & 0.70 & 11 & 3.86 & 0.007 \\
\hline HIP 37798 & 2.54 & $4082^{\mathrm{b}}$ & $\ldots$ & 1.42 & 0.70 & 9 & 3.12 & 0.005 \\
\hline HIP 38969 & 2.13 & 4761 & 0.26 & 0.40 & 0.81 & 10 & $10.96^{\mathrm{c}}$ & 0.817 \\
\hline HIP 40375 & 2.18 & 4463 & 0.03 & 0.96 & 0.71 & 33 & 6.67 & 0.009 \\
\hline HIP 40671 & 2.05 & 4612 & 0.06 & 0.46 & 0.74 & 6 & 1.95 & 0.008 \\
\hline HIP 40910 & 2.41 & $4119^{\mathrm{a}}$ & -0.06 & 1.41 & 0.68 & 21 & 9.47 & 0.665 \\
\hline HIP 41130 & 2.26 & 4410 & -0.10 & 1.10 & 0.72 & 18 & 8.46 & 0.603 \\
\hline HIP 41443 & 2.08 & 4613 & 0.01 & 0.83 & 0.74 & 9 & 6.10 & 0.014 \\
\hline HIP 42567 & 1.94 & 4648 & 0.09 & 0.71 & 0.76 & 6 & 6.00 & 0.037 \\
\hline HIP 43534 & 2.56 & $4100^{\mathrm{a}}$ & -0.13 & 1.49 & 0.64 & 14 & 6.88 & 0.006 \\
\hline HIP 43667 & 2.12 & 4554 & 0.01 & 0.51 & 0.72 & 9 & 5.24 & 0.020 \\
\hline HIP 44072 & 2.16 & 4347 & -0.42 & 0.52 & 0.71 & 5 & 3.96 & 0.020 \\
\hline HIP 45042 & 2.35 & 4476 & 0.17 & 1.27 & 0.73 & 5 & 9.94 & 0.095 \\
\hline TYC 1955-00658-1 & 2.52 & $3991^{\mathrm{a}}$ & 0.21 & 1.48 & 0.66 & 4 & 6.05 & 0.055 \\
\hline HIP 45839 & 2.12 & 4590 & 0.05 & 0.55 & 0.72 & 5 & 12.40 & 0.865 \\
\hline HIP 46343 & 2.20 & 4529 & 0.03 & 1.03 & 0.70 & 10 & 4.35 & 0.008 \\
\hline HIP 46417 & 2.28 & 4475 & -0.06 & 0.69 & 0.71 & 9 & 7.19 & 0.018 \\
\hline HIP 47201 & 2.37 & $4122^{\mathrm{a}}$ & 0.03 & 0.98 & 0.69 & 7 & 4.91 & 0.045 \\
\hline HIP 48139 & 2.09 & $4548^{\mathrm{a}}$ & 0.22 & 0.45 & 0.77 & 6 & 2.34 & 0.004 \\
\hline HIP 48411 & 2.14 & 4505 & 0.20 & 0.78 & 0.73 & 4 & 8.32 & 0.057 \\
\hline HIP 48740 & 2.22 & 4577 & 0.02 & 1.10 & 0.72 & 8 & 7.38 & 0.015 \\
\hline HIP 50960 & 2.40 & $4187^{\mathrm{a}}$ & -0.06 & 1.51 & 0.65 & 8 & 8.22 & 0.023 \\
\hline HIP 51443 & 2.16 & 4505 & -0.05 & 1.09 & 0.71 & 19 & 8.56 & 0.447 \\
\hline HIP 53327 & 2.29 & 4390 & -0.79 & 0.37 & 0.66 & 4 & 5.13 & 0.018 \\
\hline HIP 54459 & 2.26 & 4469 & -0.52 & 0.37 & 0.68 & 13 & 8.32 & 0.028 \\
\hline HIP 54651 & 2.11 & 4395 & -0.89 & 0.33 & 0.66 & 6 & 1.63 & 0.002 \\
\hline HIP 54810 & 2.09 & $4256^{\mathrm{a}}$ & 0.03 & 1.04 & 0.70 & 5 & 6.18 & 0.061 \\
\hline HIP 55507 & 2.42 & $4104^{\mathrm{a}}$ & -0.05 & 0.90 & 0.69 & 22 & $6.37^{\mathrm{c}}$ & 0.013 \\
\hline HIP 56630 & 2.67 & $3960^{\mathrm{a}}$ & -0.01 & 1.37 & 0.68 & 9 & 7.29 & 0.045 \\
\hline
\end{tabular}


Table 2

(Continued)

\begin{tabular}{|c|c|c|c|c|c|c|c|c|}
\hline Name & $V-J$ & $T_{\text {eff }}(\mathrm{K})$ & $(\mathrm{Fe} / \mathrm{H})$ & $S$ & $M\left(M_{\odot}\right)$ & $N_{\text {obs }}$ & $\mathrm{rms}\left(\mathrm{m} \mathrm{s}^{-1}\right)$ & $p^{1}$ \\
\hline HIP 57274 & 2.00 & 4641 & 0.08 & 0.39 & 0.76 & 120 & 0.00 & 1.000 \\
\hline HIP 57493 & 2.38 & $4168^{a}$ & 0.06 & 1.16 & 0.71 & 15 & 5.48 & 0.009 \\
\hline HIP 59496 & 2.41 & $4122^{\mathrm{a}}$ & -0.01 & 1.42 & 0.69 & 7 & 9.67 & 0.203 \\
\hline HIP 60633 & 2.08 & 4724 & 0.25 & 0.33 & 0.76 & 26 & 12.92 & 1.000 \\
\hline HIP 62406 & 2.38 & $4168^{a}$ & 0.31 & 1.10 & 0.68 & 39 & 8.07 & 0.118 \\
\hline HIP 62847 & 1.97 & 4726 & 0.05 & 0.66 & 0.81 & 34 & 6.03 & 0.012 \\
\hline HIP 63894 & 2.20 & $4361^{b}$ & $\cdots$ & 0.81 & 0.69 & 8 & 6.47 & 0.029 \\
\hline HIP 64048 & 2.17 & 4615 & 0.08 & 0.71 & 0.71 & 14 & 8.63 & 0.029 \\
\hline HIP 64262 & 2.03 & 4639 & -0.25 & 0.59 & 0.70 & 9 & 8.13 & 0.047 \\
\hline HIP 66074 & 2.32 & 4460 & 0.23 & 1.17 & 0.73 & 9 & 11.06 & 0.818 \\
\hline HIP 66222 & 2.71 & $3861^{\mathrm{a}}$ & -0.11 & 1.34 & 0.68 & 13 & 6.54 & 0.017 \\
\hline HIP 66283 & 1.84 & 4880 & 0.18 & 0.00 & 0.84 & 7 & 10.71 & 0.256 \\
\hline HIP 66840 & 2.47 & $4019^{a}$ & $\cdots$ & 0.00 & 0.69 & 5 & 1.96 & 0.008 \\
\hline HIP 67842 & 2.76 & $3919^{b}$ & $\ldots$ & 1.23 & 0.64 & 6 & 5.18 & 0.016 \\
\hline HIP 73427 & 2.40 & $4256^{\mathrm{a}}$ & -0.02 & 1.26 & 0.73 & 11 & 13.32 & 0.949 \\
\hline HIP 75672 & 2.45 & $4083^{a}$ & $\ldots$ & 0.00 & 0.69 & 4 & 2.67 & 0.021 \\
\hline HIP 77908 & 2.27 & 4612 & 0.28 & 0.98 & 0.74 & 5 & 2.50 & 0.008 \\
\hline HIP 78184 & 2.47 & $4168^{a}$ & $\cdots$ & 0.00 & 0.68 & 4 & 7.08 & 0.004 \\
\hline HIP 78999 & 2.05 & 4504 & 0.24 & 0.00 & 0.73 & 4 & 6.30 & 0.029 \\
\hline HIP 79698 & 2.31 & $4256^{\mathrm{a}}$ & 0.26 & 0.91 & 0.74 & 7 & 3.51 & 0.004 \\
\hline HIP 87464 & 2.17 & 4417 & -0.27 & 0.44 & 0.69 & 8 & 8.76 & 0.351 \\
\hline HIP 89087 & 2.40 & $4118^{a}$ & -0.01 & 1.38 & 0.68 & 4 & 3.48 & 0.001 \\
\hline HIP 93871 & 2.06 & $4453^{\mathrm{a}}$ & -0.47 & 0.28 & 0.71 & 4 & 3.38 & 0.001 \\
\hline HIP 97051 & 2.34 & 4320 & -0.19 & 1.10 & 0.62 & 19 & 9.10 & 0.183 \\
\hline HIP 99205 & 2.15 & 4397 & -0.18 & 0.85 & 0.64 & 6 & 6.32 & 0.021 \\
\hline HIP 99332 & 2.15 & $4400^{\mathrm{a}}$ & 0.30 & 0.73 & 0.76 & 8 & 5.82 & 0.033 \\
\hline HIP 101262 & 2.06 & 4774 & 0.19 & 1.00 & 0.73 & 4 & 9.06 & 0.146 \\
\hline HIP 102332 & 2.39 & $4202^{b}$ & $\cdots$ & 0.75 & 0.66 & 4 & 5.56 & 0.017 \\
\hline HIP 103650 & 2.10 & 4603 & -0.04 & 0.75 & 0.71 & 9 & 5.22 & 0.020 \\
\hline HIP 104092 & 2.23 & 4455 & 0.07 & 0.54 & 0.72 & 5 & 6.66 & 0.057 \\
\hline HIP 105341 & 2.55 & 4299 & -0.05 & 1.38 & 0.68 & 5 & 8.68 & 0.087 \\
\hline HIP 109980 & 2.14 & 4580 & 0.00 & 0.95 & 0.71 & 56 & 6.73 & 0.024 \\
\hline HIP 110774 & 2.14 & 4471 & -0.12 & 0.87 & 0.67 & 4 & 1.91 & 0.000 \\
\hline TYC 3995-01436-1 & 2.16 & 4634 & 0.08 & 0.89 & 0.71 & 9 & 7.20 & 0.051 \\
\hline HIP 112496 & 2.00 & 4778 & 0.04 & 0.65 & 0.75 & 5 & 7.92 & 0.059 \\
\hline HIP 112918 & 2.26 & 4434 & 0.02 & 1.20 & 0.67 & 4 & 2.80 & 0.018 \\
\hline HIP 114156 & 2.45 & 4322 & -0.02 & 1.43 & 0.66 & 4 & 3.41 & 0.025 \\
\hline HIP 115004 & 2.22 & 4684 & -0.11 & 0.83 & 0.82 & 21 & $40.79^{c}$ & 0.500 \\
\hline HIP 117197 & 2.56 & 4153 & -0.38 & 1.05 & 0.61 & 19 & 3.15 & 0.002 \\
\hline HIP 117492 & 2.10 & 4679 & 0.08 & 0.98 & 0.73 & 5 & 5.09 & 0.029 \\
\hline HIP 117559 & 2.25 & 4581 & 0.07 & 1.28 & 0.71 & 41 & 9.82 & 0.447 \\
\hline HIP 117946 & 1.95 & 4863 & 0.03 & 0.85 & 0.77 & 14 & 9.00 & 0.143 \\
\hline HIP 118261 & 1.90 & 4662 & -0.04 & 0.65 & 0.74 & 34 & 7.28 & 0.025 \\
\hline HIP 118310 & 2.24 & $4326^{\mathrm{b}}$ & $\ldots$ & 0.71 & 0.80 & 4 & 0.00 & 0.000 \\
\hline
\end{tabular}

Notes.

a Based on medium-resolution spectroscopy.

${ }^{\mathrm{b}}$ Based on $V-J$ color.

${ }^{\mathrm{c}}$ A linear trend removed.

d A parabolic trend removed.

measurements, and thus the number of degrees of freedom for a quadratic fit is $\geqslant 1$, irregular sampling means that overfitting and erroneous reduction in RV variation is possible. For example, four observations in two very closely spaced pairs cannot be reliably regressed: a significant RV offset between the pairs could be the result of a linear trend or any RV variation. To identify such cases, we computed an effective $N$ equal to the sum of normalized Voronoi-type weights $w_{i}=\left(t_{i+1}-t_{i-1}\right) / 2$ that are often assigned by regularization algorithms (Strohmer 2000). End points have weights $w_{1}=t_{2}-t_{1}$ and $w_{N}=t_{N}-t_{n-1}$. Thus,

$$
N_{\text {eff }}=\frac{3\left(t_{N}-t_{1}\right)-\left(t_{N-1}-t_{2}\right)}{2 \max \left(w_{i}\right)} .
$$

In the limit of large $N$, the effective number of points $N_{\text {eff }}$ and thus the maximum order of the polynomial that should be used in a regression approach the total time interval divided by the maximum interval between points. This is analogous to the Nyquist sampling criterion.

Doppler data sets with $\mathrm{rms}>15 \mathrm{~m} \mathrm{~s}^{-1}$ were processed in one of three ways: (1) if regressions did not significantly reduce variance ( $F$-test; $p<0.05)$, the data were analyzed as is. (2) If a regression did significantly reduce variance and $N_{\text {eff }}$ was $>3$ (or $>4$, in the case of a quadratic), the best fit is subtracted before analysis. (3) If a regression is significant but $N_{\text {eff }}$ is not sufficiently large, the star and its data were excluded from the 


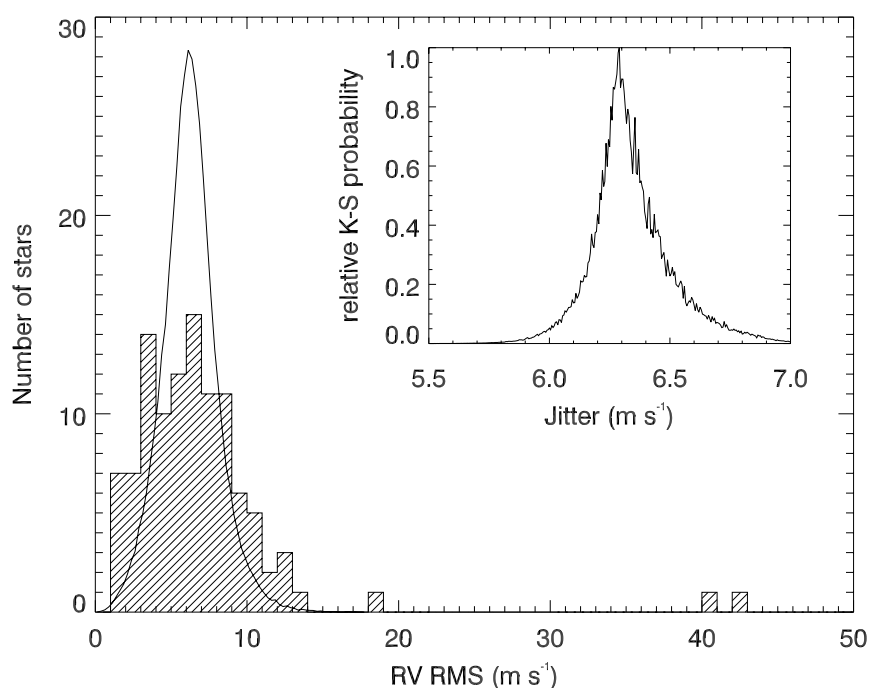

Figure 4. Distribution of adjusted radial velocity rms among $110 \mathrm{M} 2 \mathrm{~K}$ stars after excluding or adjusting case of high rms. One star with $\mathrm{rms}=65 \mathrm{~m} \mathrm{~s}^{-1}$ falls outside the plot. Systems with rms $>15 \mathrm{~m} \mathrm{~s}^{-1}$ were either excluded or significant linear/quadratic trends fitted and removed (see the text). The solid curve is the best-fit model for the resulting distribution at $\mathrm{rms}<15 \mathrm{~m} \mathrm{~s}^{-1}$ assuming pure Gaussian-distributed noise that is the sum of formal errors and an astrophysical noise term $\sigma_{0}$ that includes both stellar jitter and barycenter motion due to small planets. The value $\sigma_{0}=6.3 \mathrm{~m} \mathrm{~s}^{-1}$ which best reproduces the observed distribution was selected by maximizing the Kolmogorov-Smirnov statistic that the actual and model rms values are drawn from the same distribution (inset).

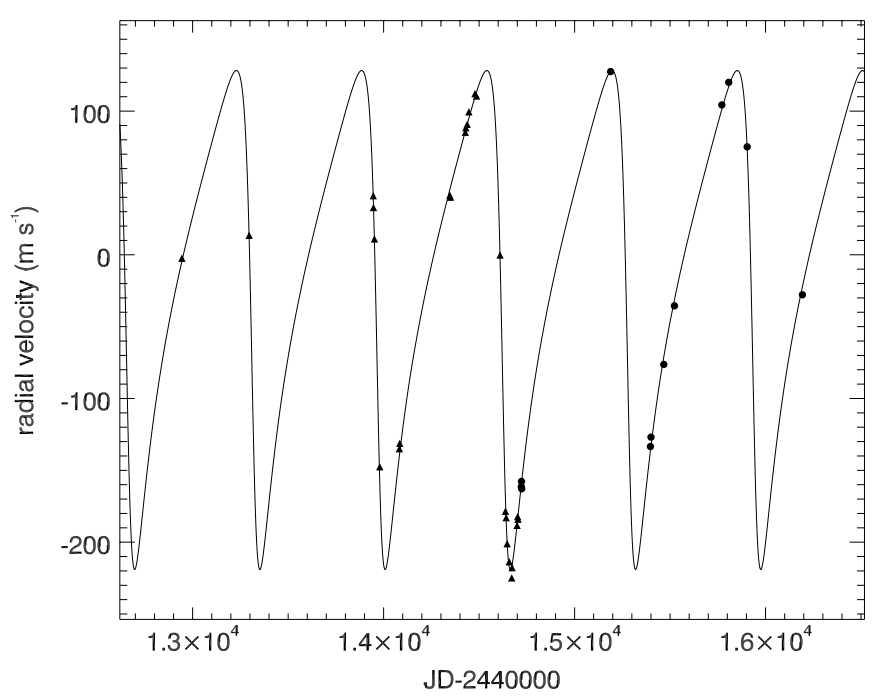

Figure 5. Thirty-eight radial velocities of HIP 2247 showing barycenter motion produced by the long-period giant planet discovered by Moutou et al. (2009). Triangles are Moutou et al. measurements with HARPS and circles are M2K measurements with Keck-HIRES. The solid line is the best-fit Keplerian orbit with $m \sin i=5.14 M_{J}, P_{K}=655.9$ days, and $e=0.543$.

analysis. We excluded 11 stars in this way, leaving 110 stars for analysis, including HIP 57274.

HIP 2247 has a long-period super-Jupiter previously identified by Moutou et al. (2009). We fit the combined HARPS and Keck-HIRES data using the RVLIN code (Wright \& Howard 2009), generating errors using 100 Monte Carlo realizations of the data by randomly reshuffling the residuals to the previous fit. We find essentially the same planetary parameters as Moutou et al. (2009), but with significantly reduced uncertainties: $M_{p} \sin i=5.14 \pm 0.02 M_{J}, P=655.90 \pm 0.22$ days, and $e=0.543 \pm 0.0011$, with a residual rms of $3.8 \mathrm{~m} \mathrm{~s}^{-1}$ (Figure 5). No significant trend was found $\left(-0.0024 \pm 0.0011 \mathrm{~m} \mathrm{~s}^{-1}\right)$. The

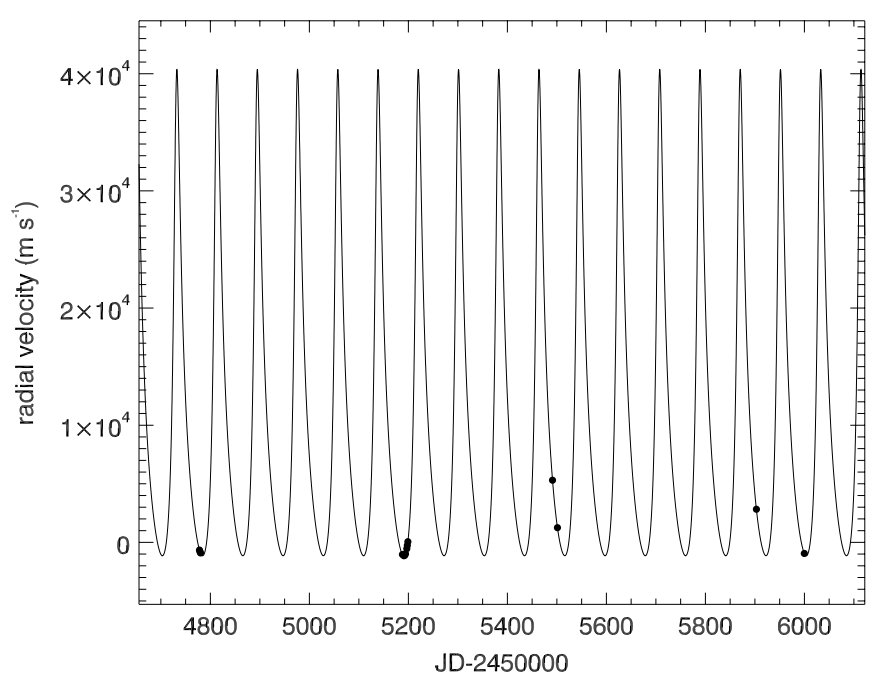

Figure 6. Fifteen $\mathrm{M} 2 \mathrm{~K}$ radial velocities of the $\mathrm{K}+\mathrm{M}$ binary star system HIP 38117. The best-fit Keplerian orbit has $P_{K}=81.28$ days and $e=0.478$.

uncertainty in $m \sin i$ does not include errors in the estimated stellar mass of $0.77 M_{\odot}$. Any giant planets with $P<245$ days can be ruled out: we include this system in our sample but consider it a definitive non-detection.

HIP 38117 exhibits RV variation consistent with the presence of a stellar-mass companion on a $81.28 \pm 0.01$ day orbit with an eccentricity of $0.478 \pm 0.012$ (Figure 6). Assuming a primary mass of $0.73 M_{\odot}$ based on the system's $V-J$ color, and assuming that the secondary contributes negligible flux, the companion's $M_{*} \sin i$ is $0.45 \pm 0.16 M_{\odot}$, i.e., this is a very late $\mathrm{K}$ or $\mathrm{M}$ dwarf. (This calculation assumes an average value of $\langle\sin i\rangle=\pi / 4$ to calculate the total system mass.) The residual rms is $3 \mathrm{~m} \mathrm{~s}^{-1}(N=15)$. As a planetary orbit with a comparable orbital period is unlikely to be stable, we follow the suit of other studies by excluding this binary system from our analysis.

\subsection{Estimation of Planet Fraction}

We estimated the fraction of stars $f$ with giant planets having $M_{P}>0.3 M_{J}$ (i.e., Saturn mass) and Keplerian orbital periods 1.7 days $<P_{K}<245$ days. The choice of outer cutoff in $P_{K}$ is based on the temporal baseline of our data-nearly all stars were monitored for at least 245 days - and motivated by the longest bin with good statistics in the analysis of Kepler planet candidates by Fressin et al. (2013). The inner cutoff corresponds to the location of the rollover in the period distribution of giant planets around Kepler stars (Howard et al. 2012). We construct and maximize a likelihood function to find the most probable value of $f$ and its uncertainty. The details of the calculations are given in the Appendix and the method is only summarized here.

A standard procedure to estimate the fraction of stars with planets is to maximize a binomial expression involving the product of detections and non-detections. However, with RV data it can be difficult or impossible to rule out all possible planets, e.g., those on face-on orbits. Thus, we replace detections and non-detections with a Bayesian statistic that is sum of the probabilities $p_{i}^{0}$ and $p_{i}^{1}$ that there are zero or one giant planets around the $i$ th star, with $1-f$ and $f$ as priors for zero or one planets, respectively,

$$
\ln \mathcal{L}=\sum_{i} \ln \left(p_{i}^{0}(1-f)+p_{i}^{1} f\right)
$$


where the sum is over all stars. This counts multi-giant planet systems once, and thus underestimates the planet occurrence (planets per star). The probabilities $p_{i}^{0}$ and $p_{i}^{1}$ are Gaussian functions of the difference between the predicted and observed RVs $v_{i j}$ and $\hat{v_{i j}}$, weighted by priors $\tilde{p}_{i}$ marginalized over all model parameters

$$
p_{i}^{n}=\left\langle\tilde{p}_{i} \exp \left[-\sum_{j} \frac{\left(v_{i j}-\hat{v}_{i j}^{n}\right)^{2}}{2 \sigma_{i j}^{2}}\right]\right\rangle,
$$

and where $\sigma_{i j}$ are the formal errors, astrophysical noise or "jitter," as well as systematic error and the contribution of small planets to motion of the star around the system's barycenter (see Section 4.1), added in quadrature. This method is analogous to the approach used in Gaidos et al. (2012) but uses the individual RV measurements, not the rms.

The Gaussian form of Equation (3) means that only the best-fit sets of parameter values (barycenter motion $v_{0}$, Keplerian period $P_{K}$, Doppler amplitude $K$, eccentricity $e$, longitude of periastron $\omega$, and time of periastron $t_{0}$ ) make significant contributions to $p^{0}$ or $p^{1}$. We used the linear dependence of the RVs on the barycenter velocity $v_{0}$ and Doppler amplitude $K$ to analytically solve for the best-fit values of these two parameters given values for the other parameters. To marginalize over planet mass we used the relation between $K$, planet mass, and orbital inclination and adopt a power-law distribution of log mass with an index $\alpha=-0.31$ (Cumming et al. 2008). (The sensitivity of our results to this value is explored in Section 4.2.) We marginalized Equation (3) over the full range of possible values of $e, \omega$, $t_{0}$, using a Rayleigh function for the prior on eccentricity (Moorhead et al. 2011) and uniform priors for $\omega$ and $t_{0}$. We further evaluated the probability over $1.7<P_{K}<245$ days at intervals of equal prior probability, assuming a power-law distribution for log period having index $\beta=0.26$ (Cumming et al. 2008). To better sample intervals of $P_{K}$ corresponding to higher probability we used each $P_{K}$ value as an initial value in a fit of a Keplerian solution to the data with the RVLIN routine (Wright \& Howard 2009). We used the fixed, best-fit values of the other parameters for the RVLIN fit, obtained an adjusted value of $P_{K}$, and then re-calculated the other parameters as described above. This procedure was repeated twice, which we found was sufficient for convergence.

We calculated $p^{0}$ and $p^{1}$ by summing over final values of all the parameters, and normalizing by $p^{0}+p^{1}$. For three stars, $p_{0}$ and $p_{1}$ were both incalculably small due to large disagreements between $v$ and $\hat{v}$ for either the zero- or one-planet models. This could be due to elevated stellar jitter or the presence of smaller planets (see below). For these stars we assigned $p_{0}=p_{1}=0.5$, i.e., the zero- and one-planet models are accepted or rejected with equal likelihood. We then evaluated Equation (2) over all possible values of $f$, and found the maximum. We calculated an approximate uncertainty by assuming asymptotic normality, iteratively fitting a parabola to the log-likelihood curve, and assigning $\sigma_{f}=1 / \sqrt{2 C}$, where $C$ is the curvature coefficient of the parabola.

Both the zero- and one-planet models do not account for barycenter motion due to the presence of other, smaller planets, as well as any sources of systematic error. As a result, for some stars both models are strongly rejected, leading to an erroneously high value of $f$. To account for this effect, we treat this barycenter motion as an additional source of uncorrelated, random RV noise or "jitter" that, along with stellar noise, can be described by a single value of $\sigma_{0}$. A value for $\sigma_{0}$ was chosen by assuming that the pronounced cluster of systems with $\mathrm{rms}<15 \mathrm{~m} \mathrm{~s}^{-1}$ (Figure 4) represents stars without giant planets, and fitting that distribution by a Monte Carlo model. We constructed 1000 artificial realizations of the data with the same number of RVs per star but drawn from a random normal distribution. The variance of this distribution was set equal to the formal measurement error and a trial value of $\sigma_{0}$ added in quadrature. We computed the rms values and comparing the distribution to the observed distribution (after subtracting any trends) with a two-sided Kolmogorov-Smirnov (K-S) test. We found that a curve with $\sigma_{0}=6.3 \mathrm{~m} \mathrm{~s}^{-1}$ (solid curve in Figure 4) maximizes the K-S probability of the Monte Carlo distribution (inset of Figure 4), and we use that value in our estimation of $f$. The poor agreement between the observed and "best-fit" distributions reflects the inability of a single value of $\sigma_{0}$ to capture the diversity of stellar RV behavior.

\section{METHODS: KEPLER SURVEY}

We compared our estimate of the fraction of $\mathrm{M} 2 \mathrm{~K}$ dwarfs with giant planets with one for late $\mathrm{K}$ dwarfs observed by Kepler. We selected Kepler targets with $1.8<V-J<2.8$, with $V$ magnitudes estimated using the relation $V=r+0.44(g-$ $r)-0.02$ (Fukugita et al. 1996). We further limited the sample to stars that had been observed in at least seven of quarters Q1-Q8. The absence of a single quarter will minimally affect the detection efficiency but is common because some stars were added after Q1 and others fall within Kepler's defunct CCD module during one of four rotations of the spacecraft. Stellar and planetary parameters of Kepler stars were estimated by fits to Dartmouth stellar models (Dotter et al. 2008) using the Bayesian procedure described in Gaidos (2013). We restricted the analysis to 6293 dwarf stars with $3900<T_{\text {eff }}<4800 \mathrm{~K}$, $\log g>4$, and $K_{P}<16$. In this sample are two giant planet candidates with $P_{K}<245$ days: KOI 1176.01 is a hot Jupiter ( $P_{K}=1.94$ days) orbiting a star with $T_{\text {eff }} \approx 4625 \mathrm{~K}$. The second (KOI 868.01) has an orbital period of 235.9 days. Another giant planet candidate (KOI 1466.01) has $P_{K}=281.6$ days and was excluded, and a fourth (KOI 1552.01) was excluded from our sample because Kepler observed it for only five of the eight quarters.

Following Mann et al. (2012), we calculated the binomial log likelihood for a flat log distribution with period and a monotonic radius distribution in the limit that the transit probability is low:

$$
\ln \mathcal{L}=\sum_{i}^{D}\left[\ln f+\ln D_{i}\left(P_{i}\right)\right]-\frac{f}{\ln \left(P_{2} / P_{1}\right)} \sum_{j}^{N D} F_{j},
$$

where the orbital period range is $P_{1}<P_{K}<P_{2}$, the two summations are over detections and non-detections, respectively, $D_{i}(P)$ is the probability of detecting a planet around the $i$ th star,

$$
F_{j}=\int_{P_{1}}^{P_{2}} D_{j}(P) d \ln P
$$

and an uninteresting constant is ignored. To compare with the M2K results, we use $P_{1}=1.7$ days and $P_{2}=245$ days.

We assumed that any transit of a giant planet in front of a late $\mathrm{K}$ dwarf will be detected. The typical transit depth is $\sim 0.02$, which is far larger than the noise: the median $3 \mathrm{hr}$ combined differential photometry precision for these stars is $1.8 \times 10^{-4}$ and the $99 \%$ value is $6.6 \times 10^{-4}$, corresponding to $\mathrm{S} / \mathrm{N}$ of 
$\sim 110$ and 30, respectively. Fressin et al. (2013) found that the recovery rate of the Kepler detection pipeline was nearly $100 \%$ for $\mathrm{S} / \mathrm{N}>16$. Thus, the detection probability is simply the geometric factor $R_{*} / a$, where $a$ is the orbital semimajor axis, and

$$
D_{j}(P)=\left(\frac{4 \pi^{2} R_{*}^{3}}{G M_{*}}\right)^{1 / 3} \frac{1+e \cos \omega}{1-e^{2}} P_{K}^{-2 / 3} .
$$

We marginalized $F$ (Equation (5)) over $e$ and $\omega$ and adopted a distribution $n(e)$ for eccentricity. Ignoring terms that do not depend on $f$, Equation (4) becomes

$$
\begin{aligned}
\ln \mathcal{L} \approx & N_{D} \ln f-0.356 f\left[\int_{0}^{1} \frac{n(e) d e}{1-e^{2}}\right]\left(\frac{P_{2}}{1 \text { day }}\right)^{-2 / 3} \\
& \times \frac{\left(P_{2} / P_{1}\right)^{2 / 3}-1}{\ln \left(P_{2} / P_{1}\right)} \sum_{j}^{N D}\left(\frac{\rho_{j}}{\rho_{\odot}}\right)^{-1 / 3}
\end{aligned}
$$

where $N_{D}$ is the number of detected planets and $\rho$ is the mean density of the star. Adopting the function for $n(e)$ in Shen \& Turner (2008), we found that the integral is only weakly dependent on the parameter $a$ in their distribution, and is $\approx 1.20$ for $a=4$. Using a Rayleigh distribution like that for the $\mathrm{M} 2 \mathrm{~K}$ analysis gives a similar value (1.08) for the integral. Because each star can be explained by more than one stellar model with probability $p$, we used a weighted mean of $\rho^{-1 / 3}$ to calculate the likelihood:

$$
\left\langle\rho^{-1 / 3}\right\rangle=\sum_{i} p_{i} \rho_{i}^{-1 / 3} / \sum_{i} p_{i},
$$

where the summation is restricted to main-sequence models, i.e., $\log g>4$.

We compared our analysis with that of Howard et al. (2012) by calculating $f$ for dwarfs with $4100<T_{\text {eff }}<4600 \mathrm{~K}$ and $4600<T_{\text {eff }}<5100 \mathrm{~K}$, and restricting the period range to 0.68 days $<P_{K}<50$ days. Our results are $0 \%$ and $0.3 \%$ for the respective $T_{\text {eff }}$ bins, compared to $0 \%$ and $2.7_{-1.4}^{+1.0} \%$ from Howard et al. (2012). Despite the same restrictions on $T_{\text {eff }}$, there are differences in the samples because we reclassified some $\mathrm{K}$ stars as giants (and any candidate giant planets as stellar companions) and we imposed a $V-J$ color cut which excludes many systems from the hotter $T_{\text {eff }}$ bin, whereas Howard et al. (2012) required $K_{P}<15$.

\section{RESULTS AND DISCUSSION}

\subsection{Fraction of Stars with Giant Planets}

Values of $p^{1}$, the probability that the RV data are consistent with the presence of a giant planet with $P_{K}<245$ days, are reported for the 110 late $\mathrm{K}$ dwarfs in Table 2 (note that $p^{0}=1-p^{1}$ ). Figure 7 shows the relative likelihood distribution of $f$ constructed from these values using Equation (2). The most probable value of $f$ is $4.0 \%$ and the uncertainty based on the assumption of asymptotic normality is $\pm 2.3 \%$. The elevated range relative to the rate of actual detections ( 1 of 110 stars) is due to the presence of several stars in our sample with significantly non-zero values of $p^{1}$. Eighteen stars have $p^{1}>0.2$ and four, excluding HIP 57274, have $p^{1}>0.9$ (Table 2). We are continuing to monitor these stars (T. S. Boyajian et al. 2013 , in preparation). If we assume that giant planets with $P_{K}<245$ days are ruled out $\left(p^{1}=0\right)$ for all stars other than HIP 57274 (the "HIP 57274 only" case in Figure 7), the most probable value of $f$ becomes $0.92 \% \pm 0.75 \%$.

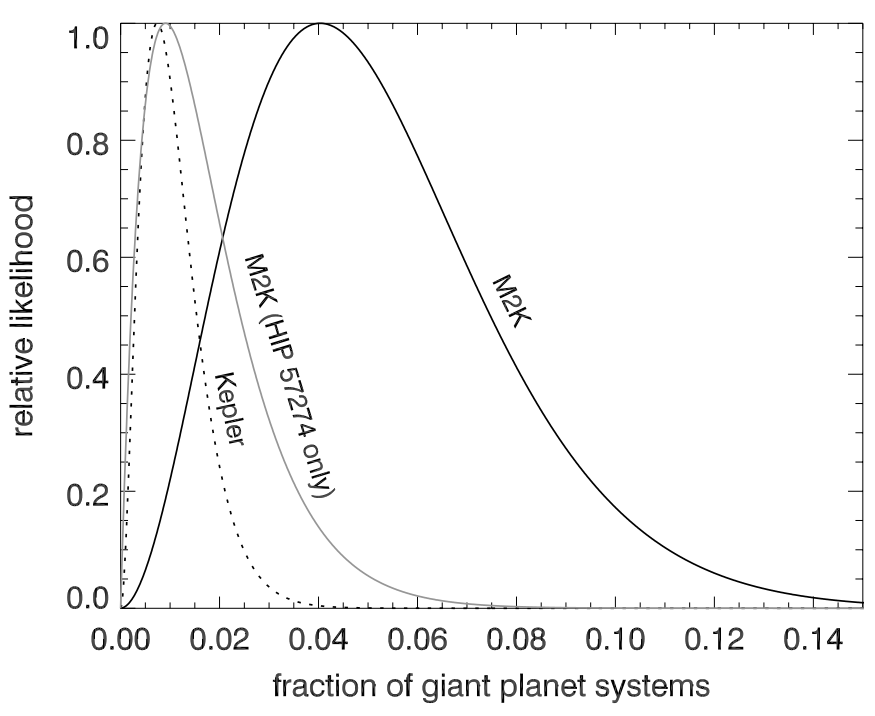

Figure 7. Probability distribution of the fraction of $110 \mathrm{M} 2 \mathrm{~K}$ stars with giant planets with $M_{p}>0.3 M_{J}$ and $P_{K}<245$ days (solid curves). The curve labeled "HIP 57274 only" assumes that such planets are ruled out around all but one of the stars: HIP 57274. The dashed line is the probability distribution of the fraction of Kepler late $\mathrm{K}$ dwarfs having giant planets with $0.7 R_{J}<R_{p}<2 R_{J}$ and $P_{K}<245$ days.

Stars may exhibit high RV variation for reasons other than the presence of giant planets with $P<245$ days. Many M2K target stars were monitored for intervals $\gg 245$ days and our RV data are sensitive to the presence of planets on wider orbits. Both Doppler and Kepler surveys find such planets, e.g., HIP 2247 and KOI 1466.01 (see below). Some stars may have a lower-mass (M dwarf) companion like that of HIP 38117 (Figure 6), but on a wider orbit. If the trend in RV produced by such a companion is not resolved because of undersampling, it will manifest itself as a high rms. Despite our precautionary elimination of stars with Ca II HK emission, some stars in our sample may have high intrinsic "jitter" from spots. Many stars in our sample have only a few Doppler observations (Figure 1), confounding these effects. Ultimately, additional observations are required to discriminate between these possibilities.

The relative likelihood distribution of $f$ for late $\mathrm{K}$ dwarfs observed by Kepler is plotted as the dashed line in Figure 7. The most likely value of $f$ is $0.7 \% \pm 0.5 \%$. Based on the two distributions, we calculate a $99 \%$ probability that the Kepler value is actually lower than the M2K value. Due to the factors discussed above, the M2K value of $4 \%$ may be an overestimate: There is a closer correspondence $(85 \%$ chance that the Kepler estimate is lower) if we rule out giant planets around all stars other than HIP 57274. Wright et al. (2012) report that the occurrence of "hot" Jupiters around the FGK stars in the CPS Doppler survey is $1.2 \%$, compared to $0.4 \%$ for Kepler (Howard et al. 2012; Fressin et al. 2013). Gaidos \& Mann (2013) proposed that the difference between the transit and Doppler results may be due to the presence of subgiants in the Kepler target catalog: planets around such stars will be more difficult to detect and more likely to experience destructive orbital decay. This explanation may be less applicable to late $\mathrm{K}$ spectral types where the giant and main-sequence branches are more distinguishable, and we consider orbits with $P_{K} \gg 10$ days on which orbital decay will be negligible. Another explanation for at least some of this difference is that the exclusion of spectroscopic and resolved binaries from the M2K sample, but not the Kepler sample, may enrich for giant planets, presuming that such binaries are less 


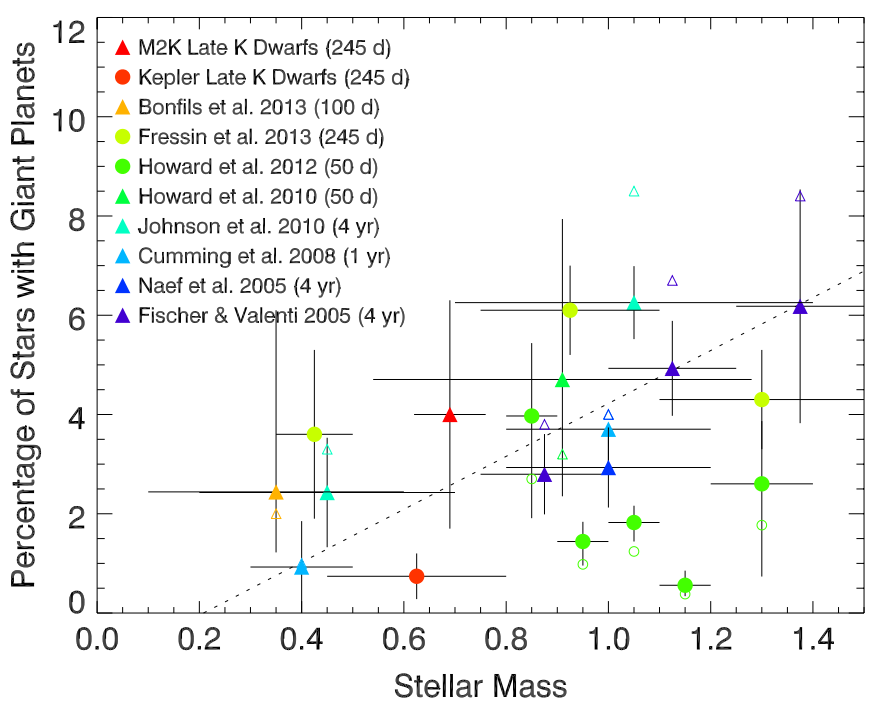

Figure 8. Adjusted percentage of stars with giant planets (Saturn mass or greater) vs. stellar mass. Points are color-coded according to their source and the symbol indicates whether the estimate is based on an RV (triangles) or transit (circles) survey. Each estimate was adjusted by the factor $\ln \left(P_{\max } / 1.7\right.$ days $) / \ln (245 / 1.7)$ to account for different choices of maximum orbital period $P_{\max }$ (see the legend). The unadjusted values are plotted as smaller open symbols of the same type and color. The range of stellar masses is in some cases approximate. The dashed line is a linear least-squares fit to the adjusted Doppler estimates.

(A color version of this figure is available in the online journal.)

likely to host planets for dynamical reasons (e.g., Thébault et al. 2006; Bonavita \& Desidera 2007; Kaib et al. 2013).

We compare our $\mathrm{M} 2 \mathrm{~K}$ and Kepler estimates of $f$ for late $\mathrm{K}$ dwarfs with previous studies for different ranges of stellar mass (Figure 8). Our estimates bridge the gap between solar-type stars (Fischer \& Valenti 2005; Cumming et al. 2008; Johnson et al. 2010; Howard et al. 2010, 2012; Fressin et al. 2013) and M dwarfs (Naef et al. 2005; Cumming et al. 2008; Johnson et al. 2010; Bonfils et al. 2013; Fressin et al. 2013). We have adjusted values by the factor $\ln \left(P_{\max } / 1.7\right.$ days $) / \ln (245 / 1.7)$ to account for differences in the maximum orbital period $P_{\max }$ of each survey, assuming a flat distribution with $\ln P_{K}$. (The adjustment is not sensitive to the exact distribution assumed.) The surveys also differ somewhat in the mass or radius ranges of objects counted as giant planets. For example, although our Kepler-based estimate of $0.7 \%$ for late K dwarfs seems much lower than those of Fressin et al. (2013) for either GK dwarfs $(6.1 \% \pm 0.9 \%)$ or $\mathrm{M}$ dwarfs $(3.6 \% \pm 1.7 \%)$, these statistics are for $P_{K}<418$ days rather than 245 days, and $R_{p}>6 R_{\oplus}$, rather than the $8 R_{\oplus}$ convention adopted here. Their overall $f$ falls to $4.1 \%$ for $P<245$ days, and our $f$ rises to $1.1 \% \pm 0.6 \%$ if we include planets with $R_{p}>6 R_{\oplus}$, bringing these two figures closer. Taken together, these estimates suggest an overall trend, perhaps linear, of increasing giant planet occurrence with stellar mass, there is not yet any indication of finer structure. A linear least-squares fit to the adjusted Doppler data yields $f(\%)=-1.11+5.33 M_{*} / M_{\odot}($ dashed line in Figure 8$)$ with weak significance ( $F$-test probability of 0.12$)$. This compilation also suggests that the deficit of giant planets around Kepler stars relative to the targets of Doppler surveys (Wright et al. 2012) depends on host star mass (Figure 8), although clearly a more homogeneous analysis of the collective data sets is needed.

A correlation between giant planets and the metallicity of the host star has been unambiguously established for solar-type stars (e.g., Fischer \& Valenti 2005), and is strongly supported by the available evidence for M dwarfs (Neves et al. 2013; Mann et al. 2013). The median metallicity of our sample of late $\mathrm{K}$ dwarfs is solar $([\mathrm{Fe} / \mathrm{H}]=0.004)$. The metallicities of HIP 57274 and HIP 2247, the two stars known to host giant planets in our sample, are 0.08 and 0.24 dex, consistent with this trend. The difference in the distributions of metallicities of stars with $p^{1}<0.1$ (median $[\mathrm{Fe} / \mathrm{H}]$ of -0.01$)$ and those with $p^{1}>0.1($ median $[\mathrm{Fe} / \mathrm{H}]$ of 0.08 ) is marginally significant (K-S probability of 0.06 ), further supporting a giant planet-metallicity relation in late $\mathrm{K}$ dwarfs. If SME overestimates the $T_{\text {eff }}$ of these stars (Figure 2), the $[\mathrm{Fe} / \mathrm{H}]$ is also overestimated by about $0.1 \mathrm{dex}$ per $100 \mathrm{~K}$.

\subsection{Sensitivity to Parameter Values}

Our estimates of $f$ may be sensitive to the values of any one of several parameters we use in our calculations (see the Appendix). These include the computational resolution $n$ with which $p_{i}^{N}$ is evaluated over ranges of the various orbital parameters, the power-law indices $\alpha$ and $\beta$ for the assumed mass and period distributions, the mean value $\bar{e}$ of the Rayleighdistributed eccentricities, and the RV jitter $\sigma_{0}$ which is assumed for each star. Due to the computational requirements of such studies, we first considered the effect on two stars, HIP 37798 and HIP 66074, with number of observations equal to the median $(N=9)$ but with the smallest $(0.005)$ and large $(0.82)$ values of $p^{1}$, respectively. Based on the outcome sensitivity of $p^{1}$ to varying parameter values, we selectively investigated the effects on our estimates of $f$.

Varying $n$ from 25 to 50 (at rapidly increasing computational cost) had a negligible effect on $p^{1}$ for HIP 37798 but decreased the value for HIP 66074 by about $13 \%$. We found that $p^{1}$ varied little for $n>50$. Thus, we re-analyzed 17 stars with $p^{1}$ values $>0.2$ (excluding HIP 57274) using $n=50$. Not all stars were re-analyzed because of the high computational cost. These $n=$ 50 values are used in the calculations of $f$ in Section 4.1. Without the substitution of high-resolution values, the most likely value of $f$ is $5.1 \% \pm 2.7 \%$.

We varied the power-law index $\alpha$ of the planet mass distribution by \pm 0.2 from its nominal value of -0.31 based on Cumming et al. (2008; see also Howard et al. 2012). $p^{1}$ increased significantly and systematically with more negative values of $\alpha$, by a factor of 3.5 for HIP 37798 and nearly 1.5 for HIP 66074 . We found that the most probable value of $f$ changed from $3 \%$ to $6.4 \%$ when we varied $\alpha$ from -0.11 to -0.51 . Also based on Cumming et al. (2008), we varied the power-law index $\beta$ of the orbital period distribution by \pm 0.1 from its nominal value of 0.26 . We found that the $p^{1}$ for HIP 37798 was essentially unchanged, while that of HIP 66074 changed by only $\pm 15 \%$. Varying $\bar{e}$ by \pm 0.1 from its nominal value of 0.225 (Moorhead et al. 2011) also had a negligible effect on the $p^{1}$ of HIP 37798 and changed that of HIP 66074 only slightly. Thus, our estimate of $f$ is not sensitive to the assumed distributions of orbital period and eccentricity, but does depend on the mass distribution. The last occurs because a steeper mass function (more negative $\alpha$ ) includes more Saturn-mass planets that could be hidden on low inclination orbits in our RV data.

Finally, we varied the value of $\sigma_{0}$ assigned to each star to account for astrophysical noise and barycenter motion induced by small planets. We considered a range of $6-6.75 \mathrm{~m} \mathrm{~s}^{-1}$ based on the K-S probability that the observed and simulated RV rms distributions are above 0.05 times the maximum value at $\sigma_{0}=6.3 \mathrm{~m} \mathrm{~s}^{-1}$ (i.e., 95\% confidence) (see Section 2.2). For both stars, values of $p^{1}$ increase significantly if $\sigma_{0}$ is decreased from its nominal value, but increased only slightly for higher $\sigma_{0}$. 
Correspondingly, $f$ increased by a factor of 1.6 for $\sigma_{0}=6 \mathrm{~m} \mathrm{~s}^{-1}$, and decreased by 0.82 for $\sigma_{0}=6.8 \mathrm{~m} \mathrm{~s}^{-1}$. A smaller $\sigma_{0}$ means that the more RV variation must be explained by the presence of giant planets, e.g., Saturn-mass planets with low orbital inclinations.

\subsection{Implications for Theory}

A correlations between the occurrence of giant planets and stellar metallicity (e.g., Fischer \& Valenti 2005; Johnson et al. 2010) has been interpreted as supporting the core accretion scenario of giant planet formation. In that scenario, growth of a sufficiently massive solid core leads to the runaway accretion of gas, but only if it occurs before the gas disk is dissipated in a few million years (Lissauer \& Stevenson 2007). In disks of higher metallicity gas, dust grains can grow, collide, and settle to the mid-plane more rapidly, thus initiating planet formation at an earlier epoch Johnson \& Li (2012). Simulations of rocky planet mass by Kokubo et al. (2006) produced a linear trend between final planet mass and initial disk mass surface density. Thus, disks around high-metallicity disks should produce larger rocky cores around which gas could accrete more quickly.

However, a trend with stellar mass, supported by our results, may require a more complex explanation. First, the dependence of disk mass on stellar mass appears to be weak (Williams \& Cieza 2011) and higher disk mass need not translate into higher mass surface density - and more massive planets (Kokubo et al. 2006) - if the radial extent of the disk is larger. Moreover, Doppler and transit surveys of FGK stars thoroughly probe orbital semimajor axes to $\lesssim 1 \mathrm{AU}$; available RV data suggest a "jump" in the population of giant planets just beyond $1 \mathrm{AU}$ (Wright \& Howard 2009) and set generous lower limits on their occurrence on much wider orbits (e.g., Wittenmyer et al. 2011). Microlensing surveys suggest that as many as a third of lensing stars (typically late $\mathrm{K}$ and $\mathrm{M}$ dwarfs) host giant planets at 1-5 AU (Mann et al. 2010; Cassan et al. 2012). If giant planet formation preferentially occurs on these orbits, the correlation with stellar mass may arise from varying efficiency of inward migration, rather than formation, of giant planets.

\subsection{On the Shoulder of Giants}

The coolest giant planet host stars in our M2K and Kepler samples are HIP 57274 and KOI 1176.01 with temperatures of $4640 \mathrm{~K}$ and $4625 \mathrm{~K}$. The only cooler $\mathrm{K}$ dwarfs hosting reported giant planets are WASP-43, HIP 70849, and WASP-80 (Table 1), but only the WASP planets are on close-in orbits. The effective temperature of WASP-43, based on the shape of the Balmer $\mathrm{H} \alpha$ line, is $4400 \mathrm{~K}$ (Hellier et al. 2011), and this is broadly consistent with the $V-J$ color of 2.4. However, this star is active and chromospheric emission may fill in and weaken the $\mathrm{H} \alpha$ line, making the temperature estimate erroneously low. An analysis of transit light curves coupled with stellar models suggests $4520 \pm 120 \mathrm{~K}$ instead (Gillon et al. 2012). The $T_{\text {eff }}$ assigned to HIP 70849 was based solely on its luminosity and a theoretical temperature-luminosity relation (Ségransan et al. 2011). WASP-80 shares spectral characteristics with both $\mathrm{K} 7$ and $\mathrm{M} 0$ dwarfs and analyses of a spectrum and infrared photometry suggests temperatures of $4145 \pm 100$ and $4020 \pm 130 \mathrm{~K}$, respectively (Triaud et al. 2013).

Depending on the properties of WASP-43 and WASP-80, these stars may bracket a $T_{\text {eff }}$ range of $4100-4600 \mathrm{~K}$ over which giant planets on close orbits have yet to be found. This could be a hint of structure, i.e., a gap or "shoulder" in the giant planet distribution with stellar mass, but any conclusion requires new surveys. Giant planets appear to orbit at wider separations around such stars (e.g., HIP 70849b and KOI 868.01), and future space-based astrometric searches with the Gaia mission (de Bruijne 2012) and microlensing surveys by Euclid (Penny et al. 2012) or the proposed WFIRST observatory (Barry et al. 2011) should reveal such planet populations in detail.

We have used the M2K and Kepler surveys to place approximate constraints on the fraction of late $\mathrm{K}$ dwarfs with giant planets, but the target catalogs are of inadequate size to address the question of any "fine structure" in the distribution of giant planets with stellar mass. The Next Generation Transit Survey (NGTS; www.ngtransits.org) will monitor 40,000 late-Gto early-M-type stars to search for "hot" Neptunes. Based on our inferred occurrence rate, we expect there to be $\sim 10$ Jupiters around these target stars; however, most of these will have orbital periods $>10$ days where the detection efficiency of a groundbased survey at a single site like NGTS is low. The Transiting Exoplanet Survey Satellite will survey $\sim 2.5$ million stars to $V=13$ (Deming et al. 2009) and, according to the TRILEGAL stellar model of the Galaxy (Girardi et al. 2005), approximately 50,000 targets will be late $\mathrm{K}$ dwarfs with $4000<T_{\text {eff }}<4800 \mathrm{~K}$. Monitoring of these should significantly improve the statistics and allow us to see further.

This research was supported by NSF Grant AST-09-08406 and NASA Grants NNX10AI90G and NNX11AC33G to E.G. Some of the data presented herein were obtained at the W. M. Keck Observatory, which is operated as a scientific partnership among the California Institute of Technology, the University of California, and the National Aeronautics and Space Administration. The Observatory was made possible by the generous financial support of the W. M. Keck Foundation. E.G. and D.F. thank the University of Hawaii and Yale Time Allocation Committees for the allocations of Keck nights used for this project. The Kepler mission is funded by the NASA Science Mission Directorate, and data were obtained from the Mukulski Archive at the Space Telescope Science Institute, funded by NASA Grant NNX09AF08G, and the NASA Exoplanet Archive at IPAC.

\section{APPENDIX}

\section{LIKELIHOOD ESTIMATION OF PLANET OCCURRENCE}

In a survey where any giant planet (within the allowed orbital period range) would be detected, and non-detections unambiguously rule out planets, the fraction of stars with giant planets $f$ can be calculated by maximizing the binomial probability distribution for $D$ detections among $N$ systems,

$$
P=\frac{N !}{D !(N-D) !} f^{D}(1-f)^{N-D} .
$$

The first factor can be ignored because it does not depend on $f$, allowing the problem to be translated into maximizing a log likelihood:

$$
\log \mathcal{L}=D \log f+(N-D) \log (1-f)
$$

However, most of our RV data are ambiguous in that they are neither detections nor can they rule out all possible giant planets. Specifically, they can only exclude planets of a certain minimum mass or minimum inclination with certain combinations of other orbital parameters. 
Equation (A2) can be generalized to $\log L=\sum_{i} \log \ell_{i}(f)$, where $\ell_{i}(f)$ is the probability that the RV data of the $i$ th star can be explained by a value of $f$. Further, the parameter $f$ describes the underlying probability distribution for the presence or absence of a giant planet, which in turn generates a model of the RV data. Using an empirical Bayesian/marginalized likelihood approach, this is expressed as a posterior probability

$$
\ell_{i}(f)=\sum_{N=0}^{1} p\left(D_{i} \mid M_{N}\right) q\left(M_{N} \mid f\right),
$$

where $p\left(D_{i} \mid M_{N}\right)$ is the probability that the $i$ th $\mathrm{RV}$ data set can be explained by a model $M_{N}$ with $N$ planets, $q\left(M_{N} \mid f\right)$ is the prior probability of $M_{N}$ given $f$, and the likelihood is marginalized over the number of planets. We seek the value of the "hyperparameter" $f$ that maximizes

$$
\log \mathcal{L}=\sum_{i} \log \left[p_{i}^{0}(1-f)+p_{i}^{1} f\right],
$$

where $p_{i}^{N}=p\left(D_{i} \mid M_{N}\right)$ marginalized over all other model parameters. Because we expect $f$ to be $\ll 1$, we neglect multiplegiant planet models.

Assuming Gaussian errors in RV,

$$
p_{i}^{n}=\exp \left[-\sum_{j} \frac{\left(v_{j}-\hat{v}_{j}^{n}\right)^{2}}{2 \sigma_{j}^{2}}\right] \tilde{p},
$$

where $v_{j}$ are the RV measurements, $\hat{v}_{j}^{n}$ are the model values for $n=0$ or 1 exoplanets, $\sigma_{j}$ are the errors, and $\tilde{p}$ represents the product of priors on the model parameters.

The RV model $\hat{v}_{j}$ of a single planet around a star depends on six parameters: barycenter velocity $v_{0}, M_{p} \sin i$, where $i$ is the inclination, orbital period $P_{K}$, eccentricity $e$, argument of periastron $\omega$, and epoch of zero true anomaly $t_{0}$. We express this as $\hat{v}_{j}=v_{0}+K g\left(t_{j}\right)$, where $K$ is the amplitude of the reflex motion,

$$
g\left(t_{j}\right) \equiv \cos \left(v_{j}\left(t_{j}\right)+\omega\right)+e \cos \omega,
$$

and $v_{j}$ is the true anomaly of the planet at epoch $t_{j}$. In the limit where $M_{p} \ll M_{*}$ the reflex amplitude is

$$
K=\frac{M_{p} \sin i}{M_{*} \sqrt{1-e^{2}}}\left(\frac{2 \pi G M_{*}}{P_{K}}\right)^{1 / 3} .
$$

The true anomaly is found by solving for the eccentric and mean anomalies $\eta$ and $\mu$,

$$
\begin{gathered}
\cos \nu=\frac{\cos \eta-e}{1-e \cos \eta} \\
\mu=\eta-e \sin \eta
\end{gathered}
$$

and

$$
\mu=\frac{2 \pi}{P}\left(t_{j}-t_{0}\right)
$$

In the single-planet model $\hat{v}_{j}=v_{0}$, which is the only parameter in this case.

A precise calculation of $p_{i}^{N}$ must marginalize over all possible parameter values weighted by $\tilde{p}$. This is computationally expensive, but if $\sigma \ll K$, Equation (3) is very sensitive to $\hat{v}_{j}$, and only best-fit parameters will make significant contributions to $p_{i}^{N}$. The best-fit value of $v_{0}$ for a star without a planet is $v_{0}^{*}=\left\langle v_{j}\right\rangle$, where

$$
\langle x\rangle \equiv \sum_{j} x_{j} \sigma_{j}^{-2} / \sum_{j} \sigma_{j}^{-2}
$$

For a star with a planet,

$$
v_{0}^{*}=\frac{\langle v\rangle\left\langle g^{2}\right\rangle-\langle v g\rangle\langle g\rangle}{\left\langle g^{2}\right\rangle-\langle g\rangle^{2}},
$$

and the best-fit $K$ is

$$
K^{*}=\frac{\langle v g\rangle-\langle v\rangle\langle g\rangle}{\left\langle g^{2}\right\rangle-\langle g\rangle^{2}} .
$$

Each possible orbit is weighted by a prior for planet mass distribution and a prior for orbital inclination (the latter is simply $\sin i$ ). However, for a given $K, M_{*}, e$, and $P_{K}$, Equation (A7) inversely relates $M_{p}$ to a unique value of $\sin i$. Thus, a marginalization over both parameters collapses to a single integral over inclination. For a power-law mass distribution with index $\alpha$

$$
\begin{aligned}
\tilde{p} & =C \int_{0}^{\pi / 2} d i \int_{M_{1}}^{M_{2}} \frac{d M_{p}}{M_{p}}\left(\frac{M_{p}}{M_{1}}\right)^{\alpha} \\
\sin i & =C \int_{0}^{\pi / 2} d i\left(\frac{M_{p} \sin i}{M_{1}}\right)^{\alpha}(\sin i)^{-\alpha} \cos i,
\end{aligned}
$$

where the normalization constant $C=-\alpha\left[1-\left(M_{1} / M_{2}\right)^{-\alpha}\right]$, and $M_{1}$ and $M_{2}$ are the lower and upper bounds to the mass range. Equation (A14) evaluates to

$$
\tilde{p}=\frac{-\alpha}{1-\alpha} \frac{M_{p} \sin i}{M_{1}} \frac{1-\left(M_{1} / M_{2}\right)^{1-\alpha}}{1-\left(M_{1} / M_{2}\right)^{-\alpha}} .
$$

The lower bound $M_{1}$ is either $0.3 M_{J}$ (the mass of Saturn) or $M_{p} \sin i$, whichever is larger, and $M_{2}=13 M_{J}$, the approximate limit for deuterium burning in brown dwarfs. $M_{P} \sin i$ is uniquely determined by $K^{*}, M_{*}, e$, and $P_{K}$. We adopt $\alpha=-0.31$ based on Cumming et al. (2008).

Equation (A15) is substituted into Equation (A5) and marginalized over $\omega \in[0,2 \pi], t_{0} \in\left[0, P_{K}\right]$, and $e \in[0,1]$. The first two are uniformly distributed, and the third is assumed to be distributed according to a Rayleigh function with a mean value of 0.225 (Moorhead et al. 2011). The only remaining parameter is orbital period $P_{K}$. We marginalize $p_{i}^{N}$ over values of $P_{K}$ drawn from a distribution $P_{1}<P_{K}<P_{2}$ in a manner that reproduces a power-law distribution with index $\beta=0.26$ (Cumming et al. 2008), with $P_{1}=1.7$ days and $P_{2}=245$ days. For better sampling of the best-fit values of $P_{K}$, we iteratively re-calculate this set of orbital periods using the Keplerian orbital fitting code RVLIN (Wright \& Howard 2009), holding other parameters fixed to their best-fit values, and iterating three time. We normalize the values of $p_{i}^{N}$ such that $p_{i}^{0}+p_{i}^{1}=1$, and then evaluate the likelihood distribution of $f$ using Equation (A3).

\section{REFERENCES}

Allard, F., Homeier, D., \& Freytag, B. 2011, in ASP Conf. Ser. 448, 16th Cambridge Workshop on Cool Stars, Stellar Systems, and the Sun, ed. C. Johns-Krull, M. K. Browning, \& A. A. West (San Francisco, CA: ASP), 91 Alsubai, K. A., Parley, N. R., Bramich, D. M., et al. 2011, MNRAS, 417, 709 Apps, K., Clubb, K. I., Fischer, D. A., et al. 2010, PASP, 122, 156 
Artigau, É., Donati, J.-F., \& Delfosse, X. 2011, in ASP Conf. Ser. 448, 16th Cambridge Workshop on Cool Stars, Stellar Systems, and the Sun, ed. C. Johns-Krull, M. K. Browning, \& A. A. West (San Francisco, CA: ASP), 771

Bailer-Jones, C. A. L. 2011, MNRAS, 411, 435

Bakos, G. Á., Hartman, J., Torres, G., et al. 2011a, ApJ, 742, 116

Bakos, G. Á., Hartman, J. D., Torres, G., et al. 2011b, in Detection and Dynamics of Transiting Exoplanets, Vol. 11, ed. F. Bouchy, R. Diaz, \& C. Moutou (Les Ulis: EDP Sciences), 1002

Baraffe, I., Chabrier, G., Allard, F., \& Hauschildt, P. H. 1998, A\&A, 337, 403

Barry, R., Kruk, J., Anderson, J., et al. 2011, Proc. SPIE, 8151, 12

Batalha, N. M., Borucki, W. J., Koch, D. G., et al. 2010, ApJL, 713, L109

Bonavita, M., \& Desidera, S. 2007, A\&A, 468, 721

Bonfils, X., Delfosse, X., Udry, S., et al. 2013, A\&A, 549, A109

Boyajian, T. S., von Braun, K., van Belle, G., et al. 2012, ApJ, 757, 112

Cassan, A., Kubas, D., Beaulieu, J.-P., et al. 2012, Natur, 481, 167

Christian, D. J., Gibson, N. P., Simpson, E. K., et al. 2009, MNRAS, 392, 1585

Cumming, A., Butler, R. P., Marcy, G. W., et al. 2008, PASP, 120, 531

de Bruijne, J. H. J. 2012, Ap\&SS, 341, 31

Deming, D., Seager, S., Winn, J., et al. 2009, PASP, 121, 952

Dotter, A., Chaboyer, B., Jevremović, D., et al. 2008, ApJS, 178, 89

Feroz, F., Balan, S. T., \& Hobson, M. P. 2011, MNRAS, 416, L104

Fischer, D. A., Gaidos, E., Howard, A. W., et al. 2012, ApJ, 745, 21

Fischer, D. A., \& Valenti, J. 2005, ApJ, 622, 1102

Fressin, F., Torres, G., Charbonneau, D., et al. 2013, ApJ, 766, 81

Fukugita, M., Ichikawa, T., Gunn, J. E., et al. 1996, AJ, 111, 1748

Gaidos, E. 2013, ApJ, 770, 90

Gaidos, E., Fischer, D. A., Mann, A. W., \& Lépine, S. 2012, ApJ, 746, 36

Gaidos, E., \& Mann, A. W. 2013, ApJ, 762, 41

Gillon, M., Triaud, A. H. M. J., Fortney, J. J., et al. 2012, A\&A, 542, A4

Girardi, L., Groenewegen, M. A. T., Hatziminaoglou, E., \& da Costa, L. 2005, A\&A, 463, 895

Gray, R. O., \& Corbally, C. J. 2009, Stellar Spectral Classification (Princeton Series in Astrophysics; Princeton, NJ: Princeton Univ. Press)

Gray, R. O., Corbally, C. J., Garrison, R. F., et al. 2006, AJ, 132, 161

Hébrard, G., Collier Cameron, A., Brown, D. J. A., et al. 2013, A\&A, 549, A134

Hébrard, G., Udry, S., Lo Curto, G., et al. 2010, A\&A, 512, A46

Hellier, C., Anderson, D. R., Collier Cameron, A., et al. 2011, A\&A, 535, L7

Henry, T. J., \& McCarthy, D. W., Jr. 1993, AJ, 106, 773

Howard, A. W., Marcy, G. W., Bryson, S. T., et al. 2012, ApJS, 201, 15

Howard, A. W., Marcy, G. W., Johnson, J. A., et al. 2010, Sci, 330, 653

Isaacson, H., \& Fischer, D. 2010, ApJ, 725, 875

Johnson, J. A., Aller, K. M., Howard, A. W., \& Crepp, J. R. 2010, PASP, 122,905
Johnson, J. L., \& Li, H. 2012, ApJ, 751, 81

Kaib, N. A., Raymond, S. N., \& Duncan, M. 2013, Natur, 493, 381

Kokubo, E., Kominami, J., \& Ida, S. 2006, ApJ, 642, 1131

Lantz, B., Aldering, G., Antilogus, P., et al. 2004, Proc. SPIE, 5249, 146

Laughlin, G., Bodenheimer, P., \& Adams, F. C. 2004, ApJL, 612, L73

Lépine, S., Hilton, E. J., Mann, A. W., et al. 2013, AJ, 145, 102

Lissauer, J. J., \& Stevenson, D. J. 2007, in Protostars and Planets V, ed. B. Reipurth, D. Jewitt, \& K. Keil (Tucson, AZ: Univ. Arizona Press), 591

Lo Curto, G., Mayor, M., Benz, W., et al. 2010, A\&A, 512, A48

Lovis, C., et al. 2011, arXiv:1107.5325

Mann, A. W., Gaidos, E., \& Gaudi, B. S. 2010, ApJ, 719, 1454

Mann, A. W., Gaidos, E., Kraus, A., \& Hilton, E. J. 2013, ApJ, 770, 43

Mann, A. W., Gaidos, E., Lépine, S., \& Hilton, E. J. 2012, ApJ, 753, 90

Moorhead, A. V., Ford, E. B., Morehead, R. C., et al. 2011, ApJS, 197,1

Moutou, C., Mayor, M., Bouchy, F., et al. 2005, A\&A, 439, 367

Moutou, C., Mayor, M., Lo Curto, G., et al. 2009, A\&A, 496, 513

Moutou, C., Mayor, M., Lo Curto, G., et al. 2011, A\&A, 527, A63

Naef, D., Mayor, M., Beuzit, J.-L., et al. 2005, in 13th Cambridge Workshop on Cool Stars, Stellar Systems and the Sun, ed. F. Favata, G. A. J. Hussain, \& B. Battrick, (ESA-SP 560), 833

Neves, V., Bonfils, X., Santos, N. C., et al. 2013, A\&A, 551, A36

Penny, M. T., et al. 2012, MNRAS, submitted (arXiv:1206.5296)

Pepe, F., Lovis, C., Ségransan, D., et al. 2011, A\&A, 534, A58

Quirrenbach, A., Amado, P. J., Seifert, W., et al. 2012, Proc. SPIE, 8446, 84460R

Schneider, J., Dedieu, C., Le Sidaner, P., Savalle, R., \& Zolotukhin, I. 2011, A\&A, 532, A79

Ségransan, D., Mayor, M., Udry, S., et al. 2011, A\&A, 535, A54

Shen, Y., \& Turner, E. L. 2008, ApJ, 685, 553

Strohmer, T. 2000, SIAM J. Sci. Comput., 22, 1160

Thébault, P., Marzari, F., \& Scholl, H. 2006, Icar, 183, 193

Torres, G., Fischer, D. A., Sozzetti, A., et al. 2012, ApJ, 757, 161

Triaud, A. H. M. J., et al. 2013, A\&A, 551, 80

Udry, S., Fischer, D., \& Queloz, D. 2007, in Protostars and Planets V, ed. B. Reipurth, D. Jewitt, \& K. Keil (Tucson, AZ: Univ. Arizona Press), 685

Valenti, J., \& Piskunov, N. 1996, A\&AS, 118, 595

Vogt, S., Allen, S. L., Bigelow, B. C., et al. 1994, Proc. SPIE, 2198, 362

Williams, J. P., \& Cieza, L. A. 2011, ARA\&A, 49, 67

Wittenmyer, R. A., Tinney, C. G., O’Toole, S. J., et al. 2011, ApJ, 727, 102

Wright, J., Marcy, G., Howard, A., et al. 2012, ApJ, 753, 160

Wright, J. T., Fakhouri, O., Marcy, G. W., et al. 2011, PASP, 123, 412

Wright, J. T., \& Howard, A. W. 2009, ApJS, 182, 205 